\title{
In vivo Assessment of Retinal Biomarkers by Hyperspectral Imaging: Early Detection of Alzheimer's Disease
}

Running Title: Hyperspectral Imaging for Early AD Diagnosis

\author{
Authors: \\ Swati S. More*1, James M. Beach ${ }^{1}$, Collin McClelland ${ }^{2}$, Ali Mokhtarzadeh ${ }^{2}$, Robert Vince*1 $^{* 1}$ \\ ${ }^{1}$ Center for Drug Design, College of Pharmacy, University of Minnesota, Minneapolis, MN 55455 \\ ${ }^{2}$ Department of Ophthalmology and Visual Neurosciences, University of Minnesota, Minneapolis, \\ MN 55455
}




\section{Figure S1}

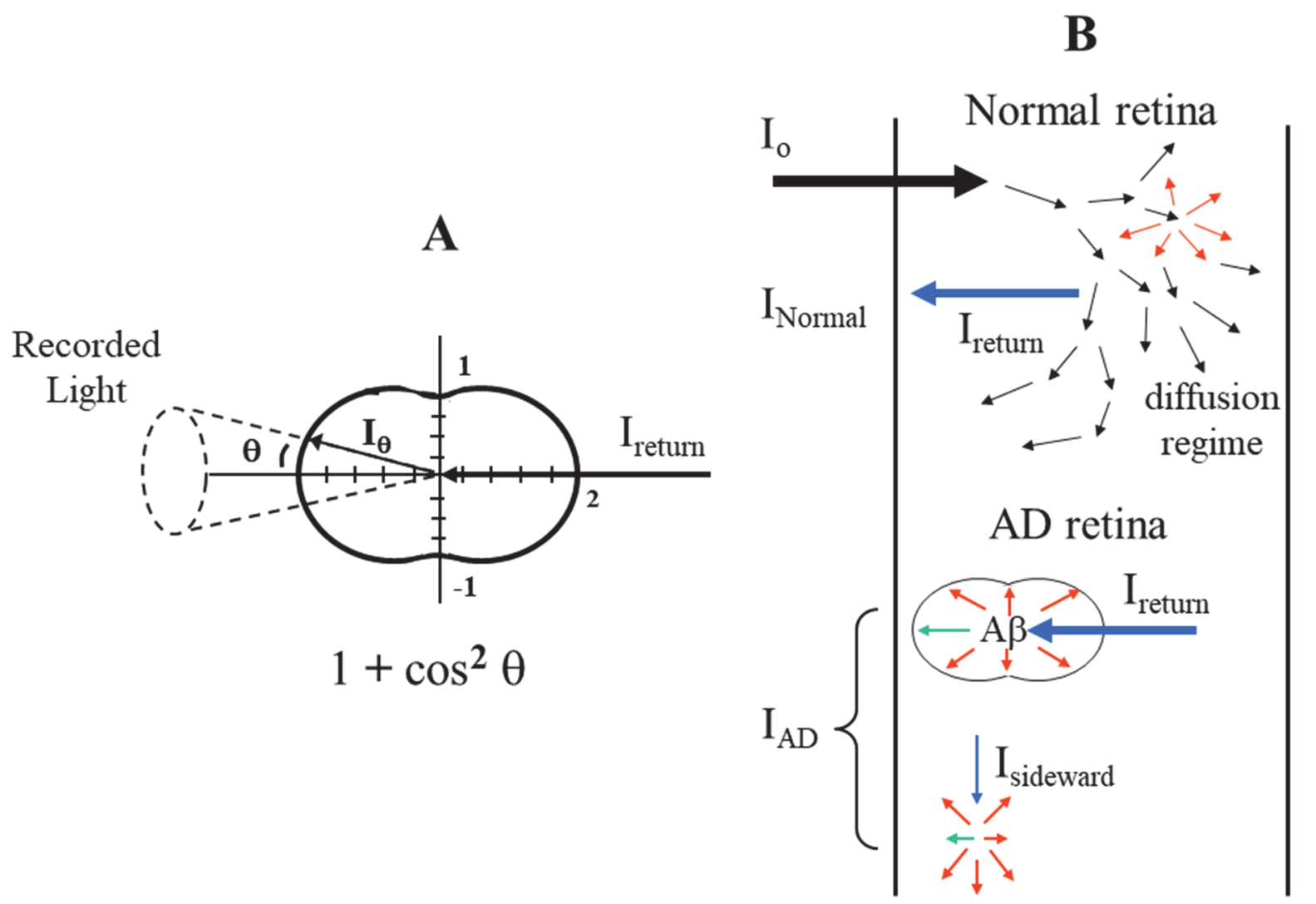

Retinal light scatter. (A) Isotropic scatter intensity profile when light interacts with an object smaller than the optical wavelength, from Eq. 2. Here light is traveling toward the detector before the interaction. (B) Top (Normal retina). Light entering the retina undergoes multiple scattering from tissue interactions. A fraction of the light returns from the retina while the remainder continues to undergo scattering and light absorption. Bottom (AD retina). Interaction of returning light with $\mathrm{A} \beta$ deflects light to the side and back causing isotropic scatter with a reduced fraction of light returning to be recorded. The reduction is dependent of size of $A \beta$ aggregates with the largest changes observed at short wavelengths. 


\section{Figure S2}
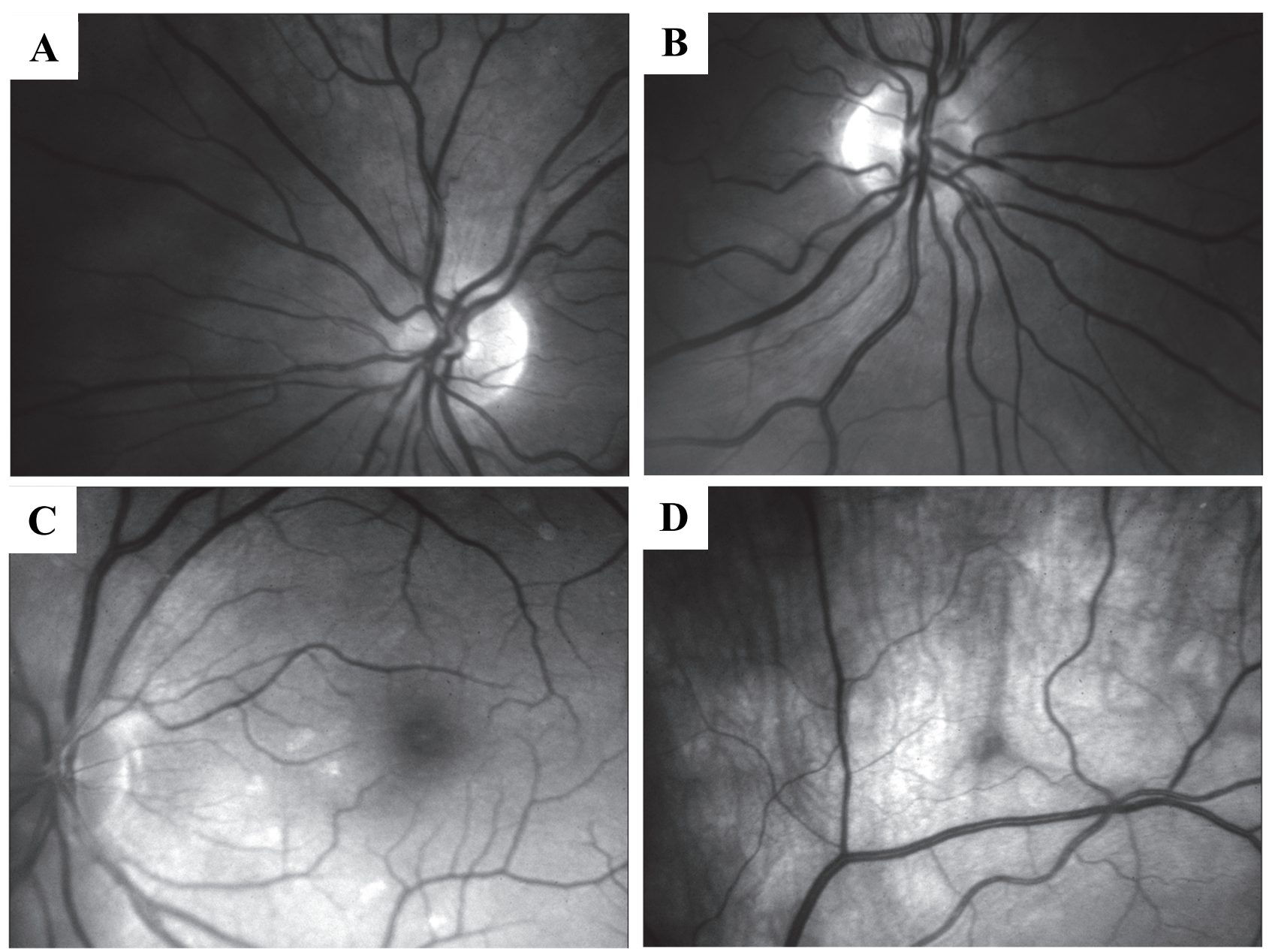

\section{D}

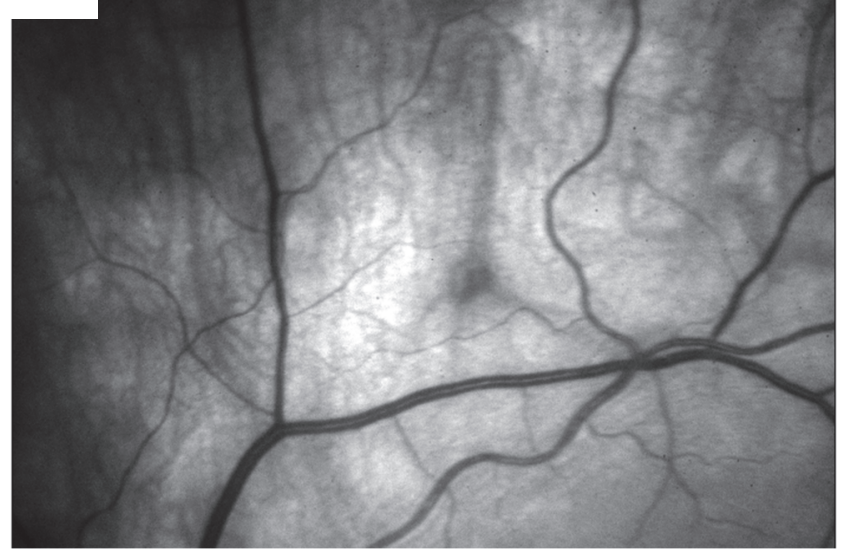

Four regions of the retina tested for spectral signature of A $\beta$ : A) optic disc; B) peripapillary retina showing nerve fiber layer running near vessels; c) macula; D) central retina. 


\section{Figure S3}

DISC

PPR

\section{MACULA}

\section{RETINA}
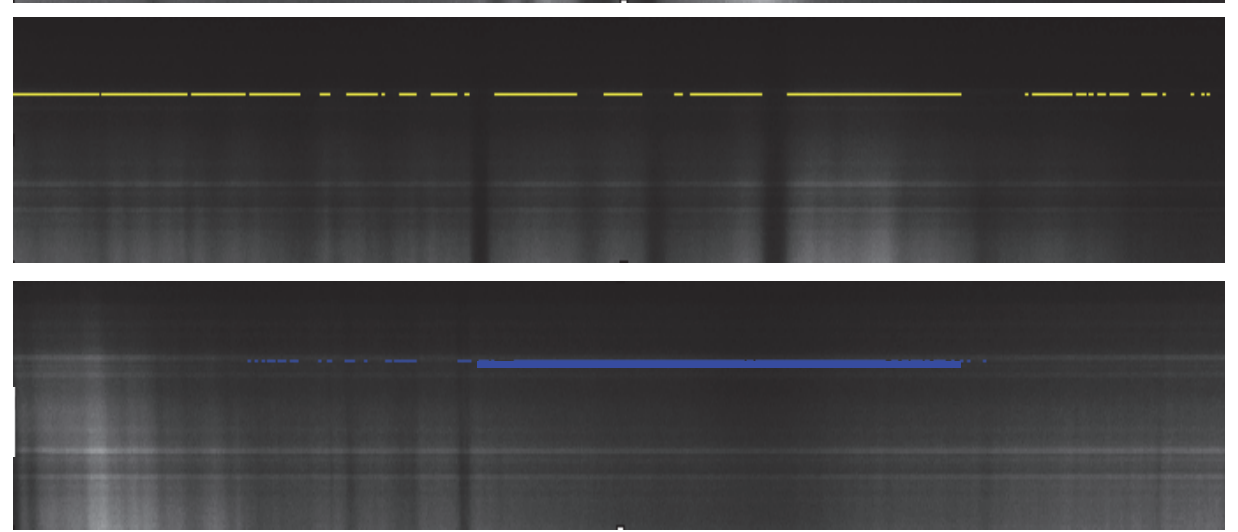
and central retina (green line) using computer-automated analysis of spectral images. Lower wavelengths $(400-550 \mathrm{~nm})$ of the full spectral range are shown. Spectra (vertical axis of for each panel) selected from spectral images obtained from each retinal area, using selection criteria described in Methods. 


\section{Figure S4}
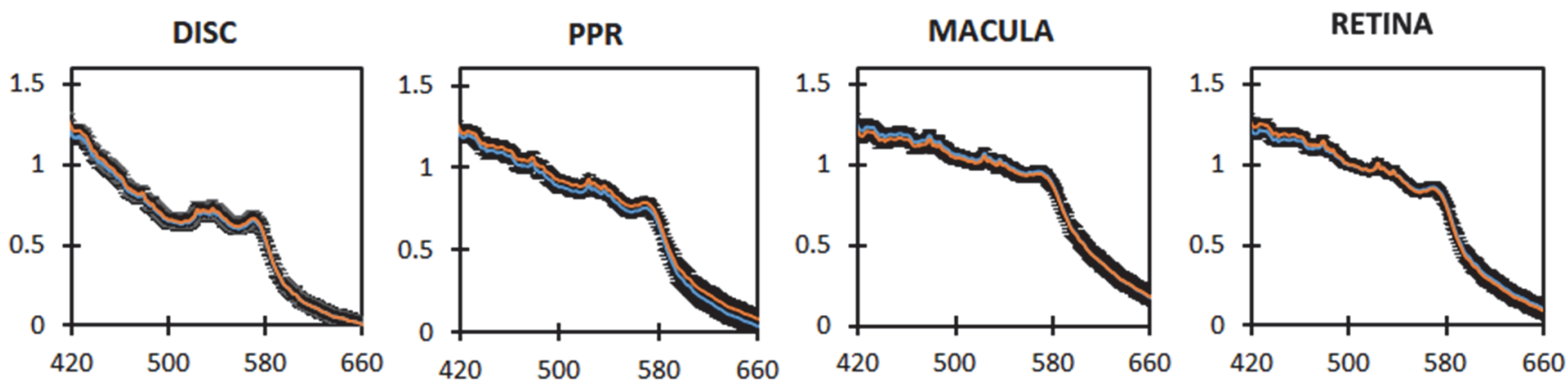

Overlap of the OD spectral profiles obtained from right eye (blue) and left eye (orange) for recordings from DISC, PPR, MACULA and RETINA areas.

TABLE S1. Statistical Analysis of AD study subgroups after normalization for age

\begin{tabular}{|c|c|c|c|c|c|c|c|c|c|}
\hline \multicolumn{10}{|c|}{ Analysis based on Cognitive Scores } \\
\hline \multirow{2}{*}{$\begin{array}{l}\text { Sub } \\
\text { group }\end{array}$} & \multirow{2}{*}{$\begin{array}{l}\text { MMSE } \\
\text { (Age) }\end{array}$} & \multicolumn{2}{|c|}{ Disc } & \multicolumn{2}{|c|}{ Peripapillary Retina } & \multicolumn{2}{|c|}{ Macula } & \multicolumn{2}{|c|}{ Retina } \\
\hline & & $\begin{array}{c}\mathrm{OD} \pm \mathrm{SE} \\
\left(\Delta \mathrm{OD}^{1}\right)\end{array}$ & $\begin{array}{l}\mathrm{P}_{\text {value }}{ }^{\text {(vs. }} \\
\text { subgroup }^{3} \text { ) }\end{array}$ & $\begin{array}{l}\mathrm{OD} \pm \mathrm{SE} \\
\left(\Delta \mathrm{OD}^{1}\right)\end{array}$ & $\begin{array}{l}\mathrm{P}_{\text {value }}{ }^{2} \text { (vs. } \\
\text { subgroup }^{3} \text {. }\end{array}$ & $\begin{array}{c}\mathrm{OD} \pm \mathrm{SE} \\
\left(\Delta \mathrm{OD}^{1}\right)\end{array}$ & $\begin{array}{l}\mathrm{P}_{\text {value }}{ }^{\text {(vs. }} \\
\text { subgroup }^{3} \text { ) }\end{array}$ & $\begin{array}{c}\mathrm{OD} \pm \mathrm{SE} \\
\left(\Delta \mathrm{OD}^{1}\right)\end{array}$ & $\begin{array}{l}\mathrm{P}_{\text {value }}{ }^{2} \text { (vs. } \\
\text { subgroup }^{3} \text { ) }\end{array}$ \\
\hline I & $\begin{array}{c}26 \\
(77.5) \\
\mathrm{N}=2\end{array}$ & $\begin{array}{c}1.567 \pm 0.006 \\
(0.318)\end{array}$ & $0.003(\mathrm{C}) *$ & $\begin{array}{c}1.441 \pm 0.052 \\
(0.192)\end{array}$ & $\begin{array}{l}0.085(\mathrm{C}) \$ \\
0.065 \text { (IV) } \\
0.150 \text { (III) } \perp\end{array}$ & $\begin{array}{c}1.412 \pm 0.008 \\
(0.248)\end{array}$ & $0.035(\mathrm{C}) *$ & $\begin{array}{c}1.529 \pm 0.002 \\
\quad(0.271)\end{array}$ & $\begin{array}{l}0.002(\mathrm{C}) * \\
0.004(\mathrm{IV}) * \\
0.042(\mathrm{III}) *\end{array}$ \\
\hline II & $\begin{array}{l}>22 \\
(73.5) \\
N=4\end{array}$ & $\begin{array}{c}1.512 \pm 0.048 \\
(0.265)\end{array}$ & $0.001(\mathrm{C}) *$ & $\begin{array}{c}1.342 \pm 0.052 \\
\quad(0.091)\end{array}$ & $0.475(\mathrm{C})$ & $\begin{array}{c}1.364 \pm 0.056 \\
(0.203)\end{array}$ & $0.022(\mathrm{C}) *$ & $\begin{array}{c}1.316 \pm 0.043 \\
(0.065)\end{array}$ & $0.663(\mathrm{C})$ \\
\hline III & $\begin{array}{c}>20 \\
(73.2) \\
N=5\end{array}$ & $\begin{array}{c}1.504 \pm 0.038 \\
(0.257)\end{array}$ & $<0.001(\mathrm{C}) *$ & $\begin{array}{c}1.261 \pm 0.041 \\
(0.009)\end{array}$ & $0.999(\mathrm{C})$ & $\begin{array}{c}1.332 \pm 0.045 \\
(0.172)\end{array}$ & $0.042(\mathrm{C}) *$ & $\begin{array}{c}1.317 \pm 0.037 \\
(0.067)\end{array}$ & $0.571(\mathrm{C})$ \\
\hline IV & $\begin{array}{c}>16 \\
(71.8) \\
N=7\end{array}$ & $\begin{array}{c}1.418 \pm 0.029 \\
(0.171)\end{array}$ & $0.013(\mathrm{C}) *$ & $\begin{array}{c}1.237 \pm 0.037 \\
(-0.015)\end{array}$ & $1.000(\mathrm{C})$ & $\begin{array}{c}1.237 \pm 0.032 \\
(0.077)\end{array}$ & $0.563(\mathrm{C})$ & $\begin{array}{c}1.261 \pm 0.032 \\
\quad(0.010)\end{array}$ & $0.999(\mathrm{C})$ \\
\hline \multicolumn{10}{|c|}{ Analysis based on Age (yr) } \\
\hline $\mathrm{V}$ & $\begin{array}{l}\leq 75 \\
\mathrm{~N}=7\end{array}$ & $\begin{array}{c}1.335 \pm 0.066 \\
(0.136)\end{array}$ & $\begin{array}{l}0.129(\mathrm{C}) \perp \\
0.188(\mathrm{VI}) \perp\end{array}$ & $\begin{array}{c}1.201 \pm 0.072 \\
(0.057)\end{array}$ & $0.297(\mathrm{C})$ & $\begin{array}{c}1.223 \pm 0.033 \\
(0.095)\end{array}$ & $0.14(\mathrm{C}) \perp$ & $\begin{array}{c}1.261 \pm 0.035 \\
(0.104)\end{array}$ & $\begin{array}{l}0.073(\mathrm{C}) \$ \\
0.069 \\
(\mathrm{VI})+\end{array}$ \\
\hline VI & $\begin{array}{l}>75 \\
\mathrm{~N}=8\end{array}$ & $\begin{array}{c}1.195 \pm 0.065 \\
(-0.005)\end{array}$ & $>1.00(\mathrm{C})$ & $\begin{array}{c}1.111 \pm 0.042 \\
(-0.031)\end{array}$ & $0.996(\mathrm{C})$ & $\begin{array}{c}1.194 \pm 0.042 \\
\quad(0.061)\end{array}$ & $0.404(\mathrm{C})$ & $\begin{array}{c}1.142 \pm 0.040 \\
\quad(-0.016)\end{array}$ & $>1.00(\mathrm{C})$ \\
\hline $\mathrm{C}$ & $\begin{array}{c}\text { Control } \\
(72.4) \\
\mathrm{N}=9\end{array}$ & $1.247 \pm 0.038$ & & $1.251 \pm 0.027$ & & $1.160 \pm 0.038$ & & $1.250 \pm 0.026$ & \\
\hline
\end{tabular}

\section{Notes}

[1] $\triangle \mathrm{OD}$ is obtained from the difference in optical densities of the AD subgroup and the control group as described in the text. All OD spectra were adjusted to zero value at $680 \mathrm{~nm}$. Values represent the OD averaged between $430 \mathrm{~nm}$ and $435 \mathrm{~nm}$.

[2] Tukey multiple comparison of subgroups, p value Confidence set at $95 \%: *$ at $90 \%: \$$ and at $80 \%: \perp$

[3] I: MSEE 26, II: MSEE > 22, III: MSEE > 20, IV: MSEE > 10, V: $\leq 75, \quad$ VI: $>75$, C: Control 